\section{P140 PREVALENCE, RISK FACTORS AND ASSOCIATION WITH DELIVERY OUTCOME OF CURABLE SEXUALLY TRANSMITTED INFECTIONS AMONG PREGNANT WOMEN IN SOUTHERN ETHIOPIA}

1,2,3 M Zenebe*, ${ }^{2} Z$ Mekonnen, ${ }^{4} E$ Loha, ${ }^{3} E$ Padalko. 'School of Medical Laboratory Sciences, Hawassa University, Ethiopia, hawassa, Ethiopia; ${ }^{2}$ School of Medical Laboratory Sciences, Jimma University, Ethiopia, Jimma, Ethiopia; ${ }^{3}$ Department of Diagnostic Sciences, Ghent University, Belgium, Ghent, Belgium; ${ }^{4}$ University of Bergen, Norway, Bergen, Norway

\subsection{6/sextrans-2021-sti.251}

Background Curable sexually transmitted infections (STIs) such as infection with Chlamydia trachomatis (C. trachomatis), Neisseria gonorrhoeae (N. gonorrhoeae), and Trichomonas vaginalis (T. vaginalis) can lead to adverse pregnancy. There are limited data on the prevalence and correlate of STI in Ethiopia, yet pregnant women are not screened for curable STI. Hence in this study, the prevalence of STIs and associated risk factors were assessed.

Methodology A cross-sectional study was conducted on consecutive women attending the delivery ward at the Hawassa comprehensive and specialized hospital. Vaginal swabs collected at the time of labor and delivery were tested for C. trachomatis, N. gonorrhoeae and T. vaginalis using GeneXpert. Study participants responded to a questionnaire about their previous and current obstetric history and socio-demographic characteristics. Possible independent factors for curable STIs were assessed by chi-square, bivariable, and multivariable logistic regression.

Results Of the 350 vaginal swabs tested, 51 (14.6\%, 95\% CI: 10.9-18.3) were positive for one or more curable STIs. The prevalence of $\mathrm{C}$. trachomatis, $\mathrm{N}$. gonorrhoeae and $\mathrm{T}$. vaginalis were $8.3 \%, 4.3 \%$, and $3.1 \%$, respectively. STIs was associated $(p<0.005)$ with the birth weight and gestational age. A 3 -fold increase in odds of acquisition STIs was documented in currently unmarried women (AOR, 3.5; 95\% CI: 1.2-10.6; p $=0.028$ ), and in younger pregnant women (AOR, 3.2; 95\% CI $1.3-7.9 ; \mathrm{p}=0.01)$. Moreover, women reporting for presence of vaginal discharge (AOR, 8.3; 95\% CI: 3.4- 20.5; p < 0.001 ) and reporting pain during urination (AOR, 6.4; 95\% CI: $2.5-16.4 ; \mathrm{p}<0.001)$ found significant associate with curable STIs.

Conlusion The higher magnitude of STIs found in this population, and the absence of symptoms in many illustrate the need for systematic follow-up during routine antenatal care primarily history taking and asking for signs and symptoms to provide early management and avoid long term sequelae.

\section{P142 LOW RETESTING AND HIGH REINFECTION RATES AMONG YOUNG PEOPLE TREATED FOR CHLAMYDIA IN AUSTRALIAN GENERAL PRACTICES}

Y Huang*, J Hocking, S Braat, J Goller. University of Melbourne, Melbourne School of Population and Global Health, Carlton, Australia

10.1136/sextrans-2021-sti.252

Background Reinfection after treatment for chlamydia is common and increases the risk of reproductive complications, particularly for women. Australian guidelines recommend retesting at 3 months after chlamydia treatment to identify reinfection. There are limited data about chlamydia retesting in Australia's mainstream primary care setting, general practice. We investigated retesting patterns among young people treated for chlamydia infection in regional Australian general practice clinics during 2010-2015.

Methods Chlamydia testing and attendance data for 16-29year-olds attending 128 regional general practices were collected for a chlamydia testing intervention trial. Rates of retesting within recommended timeframes (defined as 6-weeks6-months after an individual's first positive chlamydia test) were calculated. We examined factors associated with retesting using logistic regression models adjusting for patient sex and age-group and clustered by clinic.

Results A total of 2357 individuals (68.7\% female) with a first positive chlamydia result formed the study population. In the following 6-weeks-6-months, 26.5\% (95\% CI 24.3-28.7) reattended and were retested; $11.9 \%$ had a positive retest and positivity at retest was higher for males $(19.2 \%$, 95\% CI $14.1-$ 25.6) than females $(10 \%$, 95\%CI 9.3-15.0). A further $39.1 \%$ (95\% CI 36.1-42.2) re-attended but were not retested and $34.4 \%$ (95\% CI 31.7-37.2) did not re-attend. Multivariable analysis showed that retesting was more likely for women (adjusted odds ratio (AOR) 2.23, 95\% CI 1.79-2.79) and in intervention clinics (AOR 1.33; 95\%CI 1.07-1.64,) and that individuals aged 20-24 years were less likely (AOR 0.73; 95\% CI $0.59-0.92)$ to be retested than 16-19-year-olds.

Conclusions Rates of retesting within recommended timeframes were low and there were missed opportunities for retesting. Age and sex differences in retesting and clinics highlight the need for processes within clinics and patient focused strategies to promote reattendance and retesting. High reinfection rates further highlight the importance of retesting for timely reinfection detection and treatment.

\section{P144 MEN'S PERCEPTION OF HIV-POSITIVE STATUS DISCLOSURE IN RURAL SOUTH AFRICA}

${ }^{1,2,3}$ O Adeagbo*, ${ }^{2} \mathrm{M}$ Luthuli, ${ }^{2} \mathrm{D}$ Gumede, ${ }^{2,4} \mathrm{~J} \mathrm{Seeley,}^{2,5} \mathrm{M}$ Shahmanesh, ${ }^{1,3} \mathrm{X}$ Li, ${ }^{3} \mathrm{~K}$ Naidoo. ${ }^{1}$ University of South Carolina, Columbia, USA; ${ }^{2}$ Africa Health Research Institute, Somkhele, South Africa; ${ }^{3}$ University of Johannesburg, Johannesburg, South Africa; ${ }^{4}$ London School of Hygiene and Tropical Medicine, London, UK; ${ }^{5}$ University College London, London, UK

\subsection{6/sextrans-2021-sti.253}

Background Disclosure of an HIV-positive status has significant implications for public health outcomes, social relationships, and individual psychological and emotional well-being. The response to the disclosure is critical for future treatment adherence, feelings of stigma, future disclosure, and overall health behaviours. There is paucity of data on the impact of the reaction of the person disclosed to on men's willingness to disclose. We report on men's perception of HIV-positive status disclosure in rural KwaZulu-Natal, South Africa.

Methods Following informed consent and COVID-19 regulations, 12 telephonic in-depth interviews and 3 face-to-face group discussions (October-November 2020) were conducted with a purposive sample of men aged 23-65 years from rural KwaZulu-Natal. Themes were identified from the interview transcripts, manually coded, and analysed thematically. The study was approved by the University of KwaZulu-Natal, University of Johannesburg, and Africa Health Research Institute research ethics committee.

Results Personal characteristics, gender, HIV knowledge and HIV identity were key factors for study participants to disclose their HIV-positive status to another person. Participants reported that if that person is popular, friendly, and talkative, they will be reluctant to disclose to them fearing that they 